\title{
COVID-19 in Guizhou Province of China: Retrospective Analysis of Clinical Characteristics and Treatment Efficacy in 146 Discharged Patients
}

\section{Chunju Xue}

Guizhou Medical University

Leilei Zhou

Guizhou Medical University

Wenqing Jiang

Guizhou Medical University

Xianming Zhang ( $\square$ 13078524367@163.com )

Affiliated Hospital of Guizhou Medical University https://orcid.org/0000-0002-7503-4307

\section{Case Report}

Keywords: China, corona virus disease 2019 (COVID-19), discharge, therapeutic efficacy, ECMO

Posted Date: June 12th, 2020

DOI: https://doi.org/10.21203/rs.3.rs-33118/v1

License: (c) (i) This work is licensed under a Creative Commons Attribution 4.0 International License.

Read Full License 


\section{Abstract}

Background COVID-19 caused by severe acute respiratory syndrome coronavirus 2 (SARS-CoV-2) has hit the central China city of Wuhan since December 2019, which is highly contagious and transmitted by droplets (through cough and sneezing) and contacts. After a series of effective measures have been carried out in China, the pandemic has been reported to be aggressively contained, while more countries and regions across the world are being overwhelmed by the crisis with mounting cases. As COVID-19 is still quickly spreading globally, clinical analyses for more clinical cases are in urgent need to better understand the infection. In this study, 146 cases of COVID-19 in Guizhou province were followed up, their clinical characteristics were summarized, and therapeutic efficacy was evaluated.

Case presentation Clinical data of COVID-19 patients who were already discharged through April 15, 2020 were collected to summarize their basic conditions and evaluate therapeutic efficacy. Treatment strategies for 4 typical cases were retrospectively analyzed. Young adults were predominant cases of COVID-19 in Guizhou province. More than half of them had a clear history of recent travel to an epidemic area and family epidemic, featuring mild to moderate symptoms. The mortality was $1.4 \%$, lower than the national average. Of the cases who met the discharge criteria, $13.9 \%$ relapsed after recovery. Chest CT images indicated that few cases had different degrees of the sequelae of lung injury induced by COVID19. This could be a underlying relapse risk. The analysis of 4 cases showed that most of them had favorable prognosis.

Conclusion Most COVID-19 patients in Guizhou province are mild-to-moderate infections, with the mortality lower than the national average. However, the relapse rate after discharge is relatively high, with incomplete repair in the lung. Besides, the time window of ECMO and complications must be emphasized.

\section{Background}

COVID-19 caused by severe acute respiratory syndrome coronavirus 2 (SARS-CoV-2) has hit the central China city of Wuhan since December 2019, which is highly contagious and transmitted by droplets (through cough and sneezing) and contacts. People are generally vulnerable to the infection. The majority merely have mild to moderate symptoms with favorable prognosis, while few severe or critical cases suffer respiratory distress syndrome (RDS) complicated with sepsis and even ultimately die of multiple organ failure, which is life-threaterning to these patients ${ }^{[1,2]}$. After a series of effective measures have been carried out in China, the pandemic has been reported to be aggressively contained, while more countries and regions across the world are being overwhelmed by the crisis with mounting cases ${ }^{[3,4]}$. As of April 15,2020, the rest of the world hits grim milestone of 1,906,526 confirmed cases and 122,488 deaths $^{[5]}$. As COVID-19 is still quickly spreading globally, clinical analyses for more clinical cases are in urgent need to better understand the infection. In this study, 146 cases of COVID-19 in Guizhou province were followed up, their clinical characteristics were summarized, and therapeutic efficacy was evaluated. 


\section{Case presentation}

\subsection{Clinical data sources}

Through April 15, 2020, of the 147 confirmed cases of COVID-19 in Guizhou province, 146 were discharged and one imported case was still under treatment in hospital. Clinical data of the 146 patients who were discharged from January 31 to April 15, 2020 were collected and summarized. Their clinical characteristics and test results after discharge including SARS-CoV-2 nucleic acids, SARS-CoV-2 antibodies and chest CT images were analyzed. Treatment strategies for 4 typical cases were summarized. The study protocol was approved by the Ethics Committee of the Affiliated Hospital of Guizhou Medical University. Informed consent was obtained from each subject.

\subsection{Discharge criteria}

All included subjects met the discharge criteria according to the Scheme for Diagnosis and Treatment of 2019 Novel Coronavirus Pneumonia (The 5-7th Trial Edition) approved by National Health Commission of the People's Republic of China. The discharge criteria were as follows: (1) normal temperatures for more than 3 days; (2) significantly ameliorated respiratory symptoms; (3) significant amelioration of acute exudative inflammation indicated by lung imaging; and (4) no viral nucleic acids were found in the phlegm and nasopharyngeal swabs in two successive tests (with a sampling interval of no less than 24 h).

\subsection{Managements}

All included patients complied with the following management after discharge. Firstly, designated hospitals are in charge of contacting with grass-root hospitals in the residential districts of the patients, and timely providing the responsible residential committees and grass-root hospitals with clinical data and medical records of discharged patients. Secondly, patients were asked to wear masks and undergo a 14 day self-quarantine period of health surveillance after discharge. Patients were preferable to living in a well-ventilated room alone with separate meals in an effort to minimize close contacts with other family members. Washing hands regularly and avoiding outdoor activities were highlighted. Thirdly, patients were followed up and reexamined at hospital at 2-4 weeks after discharge. Their basic information such as name, gender, age, and discharge date was collected. Respiratory and alimentary specimens were sampled for SARS-CoV-2 nucleic acid tests (NATs). The detection of anti-SARS-CoV-2 IgM and IgG was performed. Chest CT scans were performed.

\section{Results}

\subsection{Basic clinical characteristics}

Of the included 146 cases, 144 were cured and 2 died, with a mortality rate of $1.4 \%$. There were 99 cases of mild-to-moderate infections (67.8\%), 47 cases of severe and critical illness (32.2\%), and 27 cases of underlying medical conditions (20.6\%). Fifty-one (34.9\%) reported a history of recent travel to Wuhan, 14 
(9.6\%) claimed recent travel to other cities in Hubei province, 15 (10.3\%) had traveled to other epidemic provinces, while $66(45.2 \%)$ reported no recent travel to any epidemic area. Of all subjects, 103 (70.5\%) had family epidemic of COVID-19. In our study, 74 males (50.7\%) and 72 females (49.3\%) were included, with the medium age of 40 years, ranging from 1 month 25 days to 87 years. There were 11 child patients (7.5\%) younger than 14 years and 26 patients (17.8\%) older than 60 years. As illustrated in Figure 1, young adults were predominant in the age distribution of COVID-19 in Guizhou.

The first case in Guizhou province was confirmed on January 21, 2020. Over the course of 56 days, all COVID-19 patients were discharged due March 16, 2020. On March 17, the first imported case who reported no symptoms appeared, and was confirmed on March 28 and was still under isolation and treatment. The number of daily new cases of COVID-19 and accumulated totals in Guizhou province was illustrated in Figure 2.

\subsection{SARS-CoV-2 NATs and reexaminations of anti-SARS-CoV-2 IgM and IgG after discharge}

One hundred forty-four cases (except for 2 deaths) met the discharge criteria, with an average duration of quarantine treatment of 49 days, ranging from 14-70 days. Respiratory specimens were collected from the phlegm and nasopharyngeal swabs or from anal swabs, feces and urine to detect SARS-CoV-2 nucleic acid levels. As a result, 20 cases were tested positive, with a relapse rate of 13.9凶, and 2 of them were children. As of April 15, 2020, 5 cases relapsed after discharge indicated by positive results of SARSCoV-2 NATs, with a mean age of 64 years, the average length of hospital stay of 20.8 days, the mean quarantine period of 36.5 days and the average sampling rate of 4.8 times.

Of the 20 relapsed cases, 19 were tested positive for both anti-SARS-CoV-2 IgM and IgG, with an overall sensitivity of $95 \%$; only 1 was tested negative, who still remained in quarantine for the interests of others and expected negative results of NATs and specific antibodies next time, though he could be released. Of the remaining 124 cases who showed negative results of NATs, all were tested positive for anti-SARSCoV-2 IgG and 29 were positive for both IgG and IgM.

\subsection{Reexamination of chest CT scans}

Of all included 144 cases who underwent chest CT scans at 2-4 weeks after discharged, 54 (37.5\%) presented the absence of lung inflammation without any sequela, and 90 (62.5\%) showed largely reduced lung injury which became focal inflammation with fibrosis and hyperplasia over time. Few cases had different degrees of the sequelae of lung injury, such as pleural thickening, adhesion, localized emphysema complicated with multiple bullae, focal atelectasis, focal bronchiectasis, multiple nodules (some were calcified) and enlarged mediastinal lymph nodes. Very few cases showed progressive infections, which was coincident with their relapsed SARS-CoV-2 NAT results.

\subsection{Typical cases of COVID-19}

\subsubsection{Case 1 of mild symptoms}


A 12-year-old boy was found positive for SARS-CoV-2 NATs and admitted to a designated hospital on February 9, 2020. He had traveled to Wuhan and Xiaogan in Hubei province from January 15 to 24, and denied a history of contacts with fever patients or confirmed/suspected COVID-19 patients. He returned to Guizhou on January 24, without any symptom and positive finding. During the period of self-quarantine, SARS-CoV-2 NATs revealed positive results. So he was admitted to the Affiliated Hospital of Guizhou Medical University. Laboratory examinations and the chest CT image revealed no abnormalities. He received an anti-COVID-19 strategy of ribavirin, lopinavir, ritonavir, arbidol, interferon, and lianhua qingwen granules, and was reexamined and discharged on February 17 after he met the discharge criteria.

\subsubsection{Case 2 of moderate symptoms}

A 51-year-old male who complained of fever for more than 7 days was admitted to our hospital on January 16,2020 . He went to Wuhan for a wholesale clothing business on January 5 , but did not travel to the Huanan Seafood Market, and returned on January 13. On the same day, he started to have fever (with the highest temperature of $38.5^{\circ} \mathrm{C}$ ), accompanied by cough, muscular soreness, dizziness, fatigue, a lack of appetite, and chest distress occasionally. Laboratory tests showed a low lymphocyte count and high levels of c-reactive protein (CRP) and interleukin (IL)-6. The chest CT image indicated bilateral lung infections and right-sided pleural thickening. However, antibiotic therapy showed no efficacy before positive results of SARS-CoV-2 NATs returned. The reexamination of chest CT scan showed ground-glass opacity in the lung, indicating slightly progressive infections. Given his medical history and the auxiliary examination results, he was clearly diagnosed as COVID-19. Subsequently, the man accepted an antiviral strategy including arbidol, lopinavir and ritonavir prior to an reexamination of CT scan showing significantly reduced lung inflammation and few linear opacities. He was tested negative for SARS-CoV-2 NATs twice (with a sampling interval of at least 1 day). His body temperature turned to normal for more than 3 days, and respiratory symptoms were significantly ameliorated. All this met the discharge criteria, so he was discharged on February 7. The imaging features of chest CT scans were shown in Figure 3.

\subsubsection{Case 3 of progressive severe symptoms from mild symptoms}

A 53-year-old female who was a permanent resident in Wuhan and visited her relatives in Guizhou on January 21 complained of cough with phlegm for 4 days and was admitted to our hospital. She reported a history of contacts with cough patients, and gradually developed cough with green (purulent) sputum. She reported no fever or other discomforts, but a history of hypertension and diabetes. After oral antibiotic therapy at home took no effect, she came to our hospital for diagnosis and better treatment. The physical examination showed the presence of bibasilar moist crackles. The chest CT scan revealed multiple patchy high-density shadows in the right and life lower lobes. The SARS-CoV-2 NAT for the first time showed negative results. During the hospital stay, she had fever with the highest temperature of $38^{\circ} \mathrm{C}$. The SARS-CoV-2 NAT for the second time showed positive results. The reexamination of chest CT scan indicated progressive infections. Laboratory examinations showed that the white blood cell count and lymphocyte count were $4.36 \times 10^{9} / \mathrm{L}$ and $1.05 \times 10^{9} / \mathrm{L}$. So she was confirmed as COVID-19 with mild symptoms. The patient received lopinavir and ritonavir, recombinant human interferon, lianhua qingwen 
granules, xuebijing injection for anti-inflammation therapy, and thymalfasin for immunity enhancement. However, her symptoms could not be relieved after a series of treatments, and she still complained of muscular soreness, fatigue, a lack of appetite, and diarrhea. The oxygen saturation decreased to the lowest level $86 \%$ (without oxygen inhalation). The chest CT reexamination indicated progressive infections, and laboratory examinations showed a lower lymphocyte count than before, and that the CRP level was $42.55 \mathrm{mg} / \mathrm{L}$, the IL-6 level was $34.69 \mathrm{pg} / \mathrm{ml}$, the D-dimer level was $1.61 \mu \mathrm{g} / \mathrm{ml}$ and the lactic acid level was $3.2 \mathrm{mmol} / \mathrm{L}$, which were higher than previous levels. Therefore, the patient was diagnosed as severe COVID-19 and transferred to the ICU for intensive care. She additionally and successively received oseltamivir, arbidol and ribavirin as antiretroviral therapy, methylprednisolone for antiinflammation, gamma globulin for immunity enhancement, and traditional Chinese medicine (TCM) for invigorating spleen-stomach and replenishing qi. Besides, high-flow oxygen inhalation was adopted for assisted respiration, and symptomatic and supportive treatment was employed. After the mentioned treatments, her symptoms were ameliorated and laboratory indices were reduced. The chest CT reexamination showed reduced inflammation, and SARS-CoV-2 NATs returned negative twice. All this met the discharge criteria, so she was discharged on February 17. The overall length of hospital stay was 18 days. Her imaging features of chest CT scans were shown in Figure 4.

\subsubsection{Case 4: a critical patient who finally died even after plasmapheresis and ECMO therapy}

A 33-year-old male who had a trip to Wuhan on January 15 began to have fever and cough along with fatigue and muscular soreness after his return on January 21. He complained of cough with fever for more than 10 days and was admitted to a local hospital. He had a history of hypertension. After the young man visited the local hospital in Guizhou, the chest CT scan was performed on January 22 and showed ground-glass opacity and exudative lesions in both lungs. The SARS-CoV-2 NAT was positive. So he was diagnosed as COVID-19 and received lopinavir and ritonavir for antiviral treatment and other symptomatic treatments. On the contrary, his conditions were worsening, and he began to have hyperpyrexia (the highest temperature of $39^{\circ} \mathrm{C}$ ) with diarrhea, dyspnea (a respiratory rate of 43 breaths/min) and a decreasing oxygen saturation to $76 \%$. To rescue him from danger, on January 31 , he was transferred to the ICU with an negative pressure environment at our hospital for intensive care. Laboratory examination results returned as follows: WBC of $22.15 \times 10^{9} / \mathrm{L}$, the proportion of neutrophils of $91.7 \%$, the lymphocyte count of $1.12 \times 10^{9} / \mathrm{L}$, the procalcitonin level of $2.83 \mathrm{ng} / \mathrm{ml}$, the IL- 6 level of 23.01 $\mathrm{pg} / \mathrm{ml}$, the CRP level of $246.2 \mathrm{mg} / \mathrm{L}$, the CK level of $439.32 \mathrm{U} / \mathrm{L}$, the CK-MB level of $49.0 \mathrm{U} / \mathrm{L}$, the LDH level of $855.0 \mathrm{U} / \mathrm{L}$ and the D-dimer level of $1.31 \mu \mathrm{g} / \mathrm{ml}$. The bedside chest CT imaging was performed on January 31 and showed large-scare patchy and high-density shadows with unclear borders and obscure lung markings. He received lopinavir, ritonavir, arbidol and $a$-interferon for antiviral treatment, meropenem combined with moxifloxacin for anti-infection treatment, methylprednisolone, and immune globulin and xuebijing injection for anti-inflammatory treatment, along with albumin for the correction of hypoalbuminemia, as well as sedation and analgesia. However, even with the trachea cannula and assisted invasive mechanical ventilation (inhalation of pure oxygen), his oxygen saturation (65-70\%) could not be increased. He then had the presences of type I respiratory failure (T1RF), acute respiratory 
distress syndrome (ARDS), and multiple organ dysfunction syndrome (MODS) especially in the kidney, lungs and peripheral circulation system over time. After discussions with experts in our hospital, extracorporeal membrane oxygenatio (ECMO) was used (pulse amplitude range 3500-4100 rpm, 4.0-5.3 $\mathrm{L} / \mathrm{min}, \mathrm{Fi02} 100 \%$ ), along with plasmapheresis. With these treatments, his symptoms were improved and his oxygen saturation was increased (92-99\%). Laboratory test results returned and indicated decreased levels of indices and the recovery of renal function. The bedside chest CT scan performed on February 3 showed reduced exudation in both lungs. The young man was in better conditions. He received blood transfusion for correcting anemia. However, the blood test on February 4 showed that the Hb level was 61 $\mathrm{g} / \mathrm{L}$, which was decreasing. Then massive pleural effusion was found during careful examinations, so the thoracic close drainage was performed and a large volume of bloody pleural fluid was removed. This was considered to be induced by complications of ECMO treatment. Afterward, the patient appeared unstable circulation and haemorrhagic shock. After the drainage tube was clamped, he received the transfusion of leukocyte-depleted red blood cells for blood supplement, and of fresh apheresis platelets, fresh-frozen plasma, cryoprecipitates, factor VII and fibrinogen complexes for improving the coagulation function and reducing bleeding. Even with these proactive managements, his vital signs were still unstable. After an emergent meeting with experts, bedside thoracoscopic exploration and hemostatic therapy were carried out, simultaneously with emergency rescue and preparations for surgery. Nonetheless, cardiac arrest occurred in the young adult at 01:50 a.m. on February 5. The heart rate and blood pressure could not be measured, and main pulse disappeared. The ECG showed a flat line. The young man was declared clinically dead even after a series of rescue measures. The causes of death were as follows: (1) critical COVID-19, (2) severe ARDS, (3) acute respiratory failure (severe), (4) hemorrhagic pleural effusion on the left side, (5) hypovolemic shock, (6) MODS in the coagulation, respiratory and circulation systems and the kidney, and (7) severe anemia. The imaging features of chest CT scans were shown in Figure 5.

\section{Discussion}

As with our analysis of epidemiological data, more than half of confirmed cases have a definite history of recent travel to an epidemic area or family epidemic. There is no gender-specific prevalence, and people are generally vulnerable to the infection. In view of age-specific prevalence, young adults are more prevalent as they have wide social connections and frequent contacts with people, who are the mainstream of population movement. So this is the leading cause of the fast spread of this infection. Both daily new cases and accumulative totals in Guizhou are fewer than those in other regions, cities and provinces in China. This may attribute to the advantage that Guizhou is located in the Yungui Plateau, a relatively undeveloped region far from the epidemic center Wuhan. Even so, the local government and medical staffs have to maintain sharp vigilance in imported cases, and residents must proactively protect themselves.

So far, while tens of thousands COVID-19 cases across the world have been cured and discharged, few of them still show positive results of SARS-CoV-2 NATs. So the relapse issue has been a health concern for many. In Guizhou, the relapse rate reaches $13.9 \unrhd$ of the discharged totals. This may attribute to the remaining SARS-CoV-2 in the lung or other tissues when patients' clinical symptoms and imaging 
manifestations are ameliorated and respiratory NAT results become negative. Recent studies rarely report histological and pathological characteristics in patients with mild infections and cured patients. In a latest study reporting the postmortem pathology for an old patient with mild COVID-19 who has unexpectedly died of heart disease after he has met the criteria of three continuous negative NAT results and has been discharged, the pathological experiments for the lung, liver, heart, intestines and skin slices show that SARS-CoV-2 still remains in the lung. This indicates that the virus can still retain in the lung when it cannot be measured in nasopharyngeal swabs. So we forcefully suggest that the inclusion of NATs of bronchoalveolar lavage fluid in the discharge criteria and a prolonged quarantine time.

Of those who have met the discharge criteria in Guizhou province, cases showing the disappearance of lung injury in CT images only account for less than $50 \%$, leaving the majority have partially reduced lung injury in CT images. The incompletely reduced lung inflammation can be localized over time, showing the tendency of fibrosis and hyperplasia. Few cases may have different degrees of the sequelae of lung injury, such as pleural thickening, adhesion, and localized emphysema or with multiple bullae, which can affect the quality of life and can even become an underlying relapse risk in the future.

Patients with mild-to-moderate COVID-19 are predominant in Guizhou province, of whom children have mild infections and old adults with severe underlying diseases often have severe and critical infections. The proportion of severe and critical cases is slightly higher than the national average, however, the mortality rate is lower than the national average $\mathrm{e}^{[6-7]}$. Older adults and people who have underlying medical conditions can be at higher risk for severe illness and progression of COVID-19. Few patients with mild or moderate illness have progressive infection after admission, which even develops into severe or critical illness afterward. Therefore, early identification and intervention is a pressing issue. A previous study ${ }^{[8]}$ indicates that any individual who has the following conditions is more likely to have progression of COVID-19: (1) people who are older than 60 years (2) complicated with severe chronic underlying diseases (e.g., hypertension, diabetes, coronary heart disease, malignant tumors, structural lung diseases, immunosuppression, etc.), and (3) who have continuous reduced lymphocyte counts, (4) fast spread of lung infections indicated by chest CT findings and (5) continuous hyperpyrexia, and (6) whose oxygenation index cannot be significantly improved after high-flow nasal cannula (HFNC) oxygen therapy, with progressive increases in (7) the serum lactic acid level and (8) the D-dimer level. As a result of this summary, we should pay attention and quickly respond to these manifestations early on in clinical practice.

With respect to strategies for critical patients, the time window of ECMO and the surveillance of complications induced by ECMO are of great importance. Evidences ${ }^{[9.10]}$ show that the common complications of ECMO encompass mediastinal hemorrhage, intracranial hemorrhage, infections, limb ischemia, hepatic and renal impairments, etc., of which the most critical is hemorrhage. Among the 4 typical cases, hemorrhagic pleural effusion has been considered to be coagulation failure induced by ECMO. Moreover, SARS-CoV-2 infection can also cause coagulation disorders, which should be differentiated as their treatments are distinct with specific therapeutic targets. 


\section{Conclusions}

In summary, the mortality of COVID-19 patients in Guizhou province is lower than the national average, but with a higher relapse rate. This may attribute to the remaining SARS-CoV-2 in the lung or other tissues after patients' clinical symptoms and imaging manifestations are ameliorated and respiratory NATs are tested negative. Old age and underlying diseases are two risk factors of progressive infection of COVID19. In terms of the treatment for critical patients, the time window of ECMO application and complication surveillance are of great importance.

\section{Abbreviations}

COVID-19 : corona virus disease 2019

ECMO : extracorporeal membrane oxygenation

RDS : respiratory distress syndrome

NAT : nucleic acid tests

CRP : c-reactive protein

TCM: Traditional Chinese Medicine

T1RF : type I respiratory failure

ARDS : acute respiratory distress syndrome

MODS : multiple organ dysfunction syndrome

HFNC : high-flow nasal cannula

\section{Declarations}

\section{Ethics approval and consent to participate}

The medical research protocol was approved by the Ethics Committee of the Affiliated Hospital of Guizhou Medical University.

\section{Consent to publication}

During their stay and treatment in our department, the patients signed the standard required statement, confirming their informed consent for our team to present, report and publish their anonymous individual patient data.

\section{Availability of data and material}

The datasets used and/or analysed during the current study are available from the corresponding author on reasonable request.

\section{Competing interests}

The authors declare that they have no competing interests. 


\section{Funding}

This work was funded by National Natural Scientific Foundation of China (Grant No. 81660018).The funding body play an important role in design of the study and collection, analysis, and interpretation of data and in writing the manuscript.

\section{Authors' contributions}

CJX took responsibility for the integrity of the data and the accuracy of the data analysis. LLZ and WQJ had full access to all data in the study. XMZ contributed to the review and approved the final version.

\section{Acknowledgements}

Not applicable

\section{Authors' information}

The first author Chunju Xue is a doctoral candidate.

The corresponding author Xianming Zhang is the director of respiratory department, chief physician, professor, supervisor of doctoral and master students in affiliated hospital of guizhou medical university. He is a senior visiting scholar in Heidelberg university, Germany and ancona university, Italy. Currently, he is the vice chairman of the branch of tuberculosis of guizhou medical association and the vice chairman of integrated traditional Chinese and western medicine of guizhou province. In the past two years, he has successfully applied for and presided over a number of projects such as the national natural science foundation of China, applied for 6 utility model patents as the first inventor, and participated in the compilation of 3 monographs. He was selected as a "thousand-level" innovative talent in guizhou province in 2017.

\section{References}

1. Zhu N, Zhang D, Wang W, et al. A novel coronavirus from patients with pneumonia in China, 2019. [J]. N Engl J Med. 2020.382(8):727-733. doi: 10.1056/NEJMoa2001017.

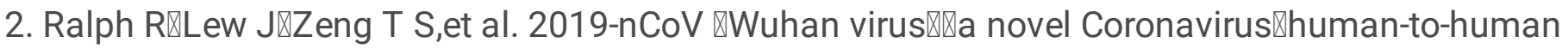
transmission囚travel-related cases \and vaccine readiness[J]. J Infect Dev Ctries. 2020.14囚1ه囚3-17.

3. Paules $\mathrm{Cl}$, Marston HD, Fauci AS. Coronavirus infections-more than just the common cold [J]. JAMA. 2020. doi:10.1001/jama.2020.0757.

4. Hui DS, I Azhar E, Madani TA, et al. The continuing 2019-nCoV epidemic threat of novel coronaviruses to global health - The latest 2019 novel coronavirus outbreak in Wuhan, China[J]. Int J Infect Dis. 2020: 264-266. doi: 10.1016/j.ijid.2020.01.009.

5. WHO main website. https://www.who.int (accessed April 15th, 2020). 
6. Yao Xiao-Hong,He Zhi-Cheng,Li Ting-Yuan, et al. Pathological evidence for residual SARS-CoV-2 in pulmonary tissues of a ready-for-discharge patient.[J]. Cell research. 2020. doi:10.1038/s41422-0200318-5.

7. Huang C, Wang Y, Li X, et al. Clinical features of patients infected with 2019 novel coronavirus in Wuhan, China [J]. Lancet. 2020. 395(10223): 497-506. doi:10.1016-S0140-6736(20)30183-5.

8. Chen N, Zhou M, Dong X, et al. Epidemiological and clinical characteristics of 99 cases of 2019 novel coronavirus pneumonia in Wuhan, China: a descriptive study[J]. Lancet, 395(10223): 507-513. doi:10.1016-S0140-6736(20)30211-7.

9. Wang G, Jin X. The progress of 2019 novel coronavirus event in China[J]. J Med Virol. 2020. doi:10.1002/jmv.25705.

10. Longcun. Expert consensus on support of extracorporeal membrane oxygenation [J].Chinese Journal of Extracorporeal Circulation. 2014.12(02):65-67.doi囚10.13498/j.cnki.chin.j.ecc.2014.02.01.

11. Michael S. Mulvihill A. David N, et al. Extracorporeal membrane oxygenation following lung transplantation: indications and survival[J]. Journal of Heart and Lung Transplantation. 2017. doi:10.1016/j.healun.2017.06.01.

\section{Figures}

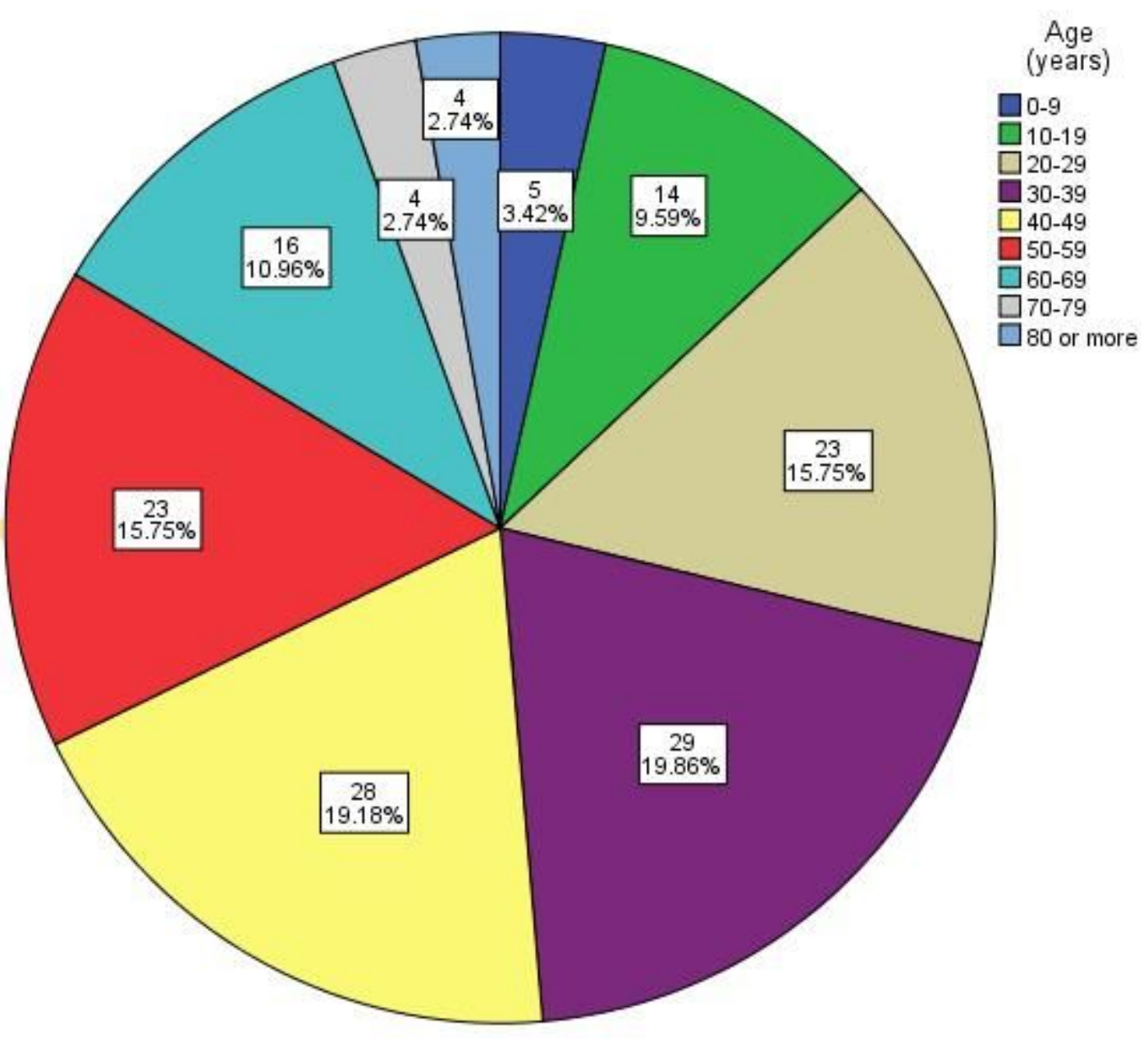


Figure 1

Age distribution of patients with COVID-19 in Guizhou Province.Data are presented as n/N (\%).
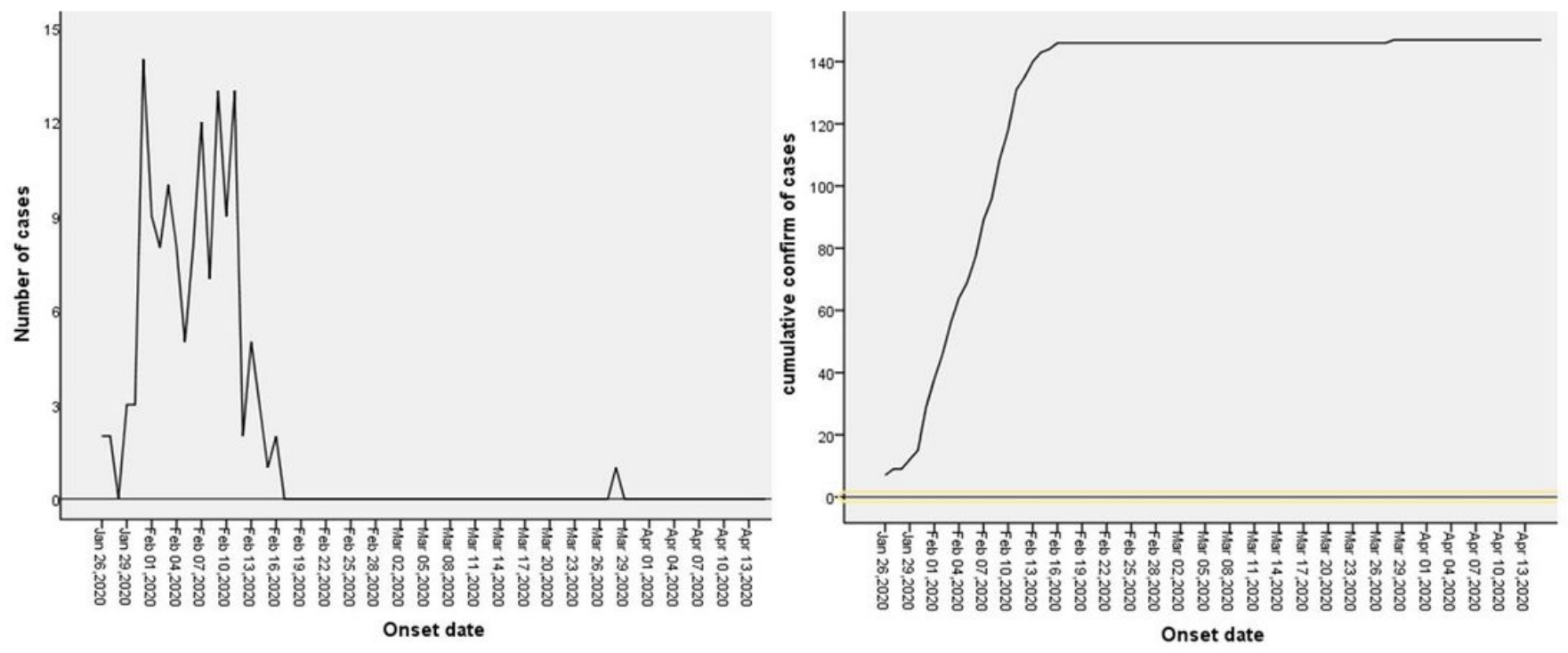

Figure 2

The number of daily new cases of COVID-19 and accumulated totals in Guizhou province. Data are presented as the number of cases. 


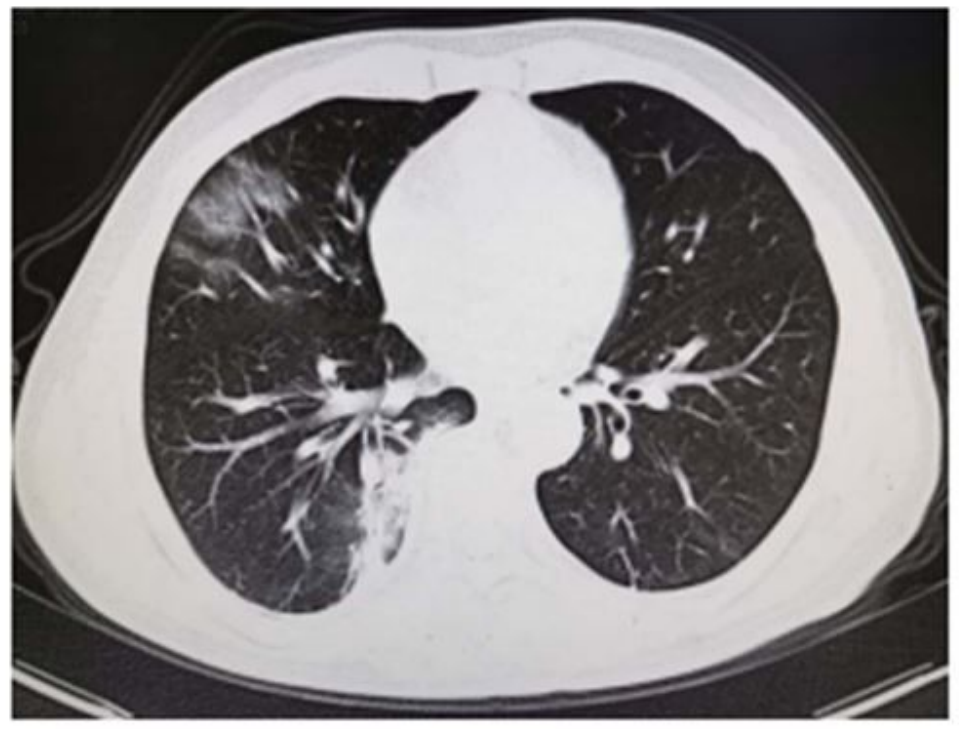

A $2020-01-16$

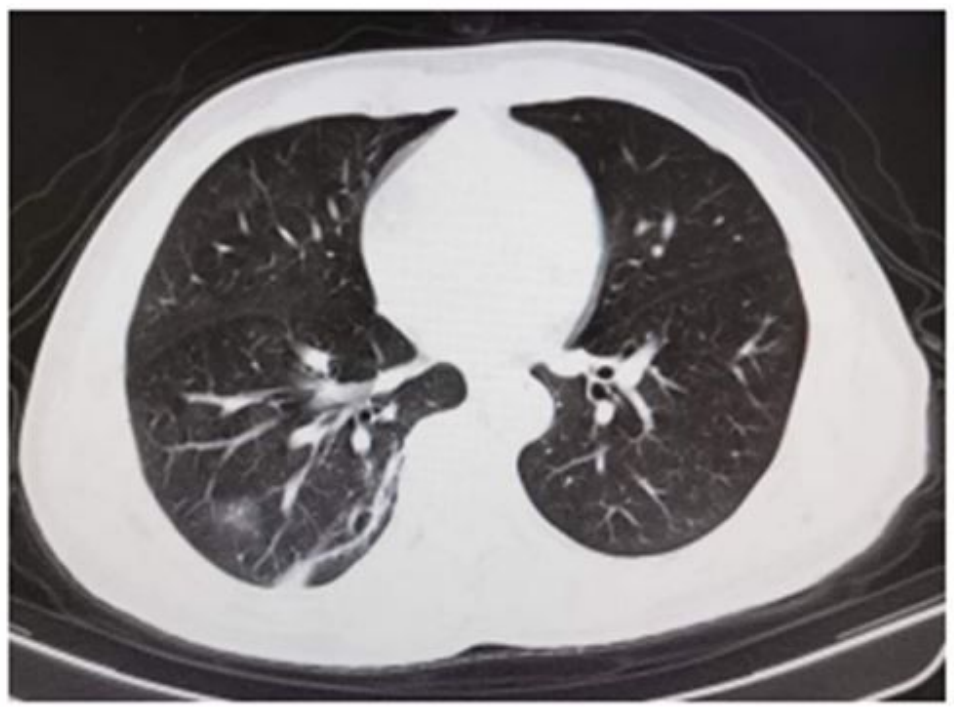

C 2020-01-27

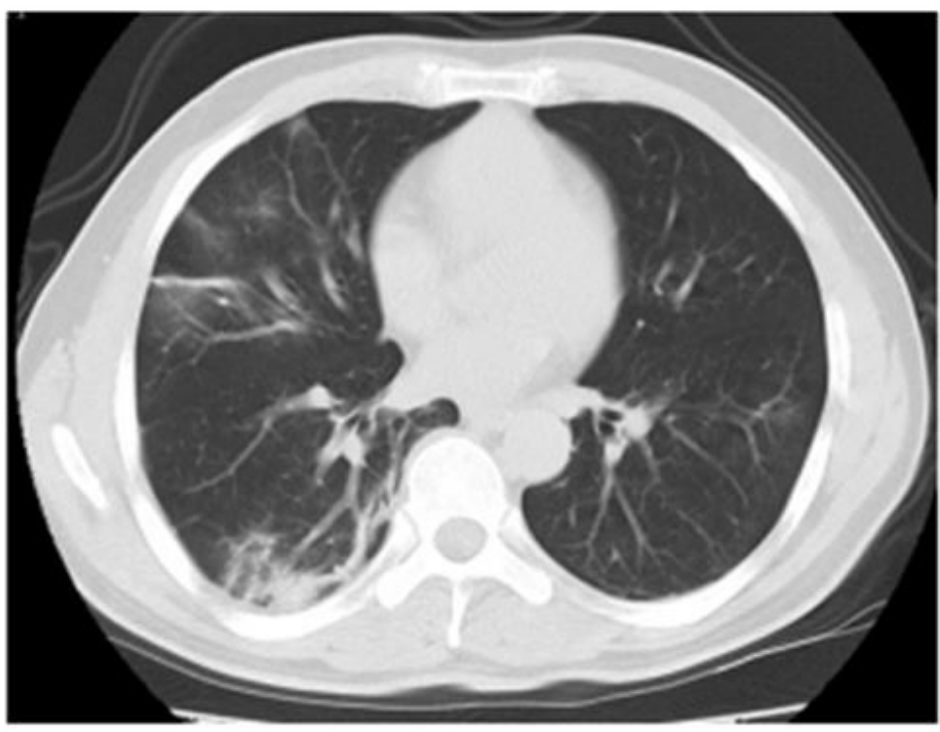

B 2020-01-20

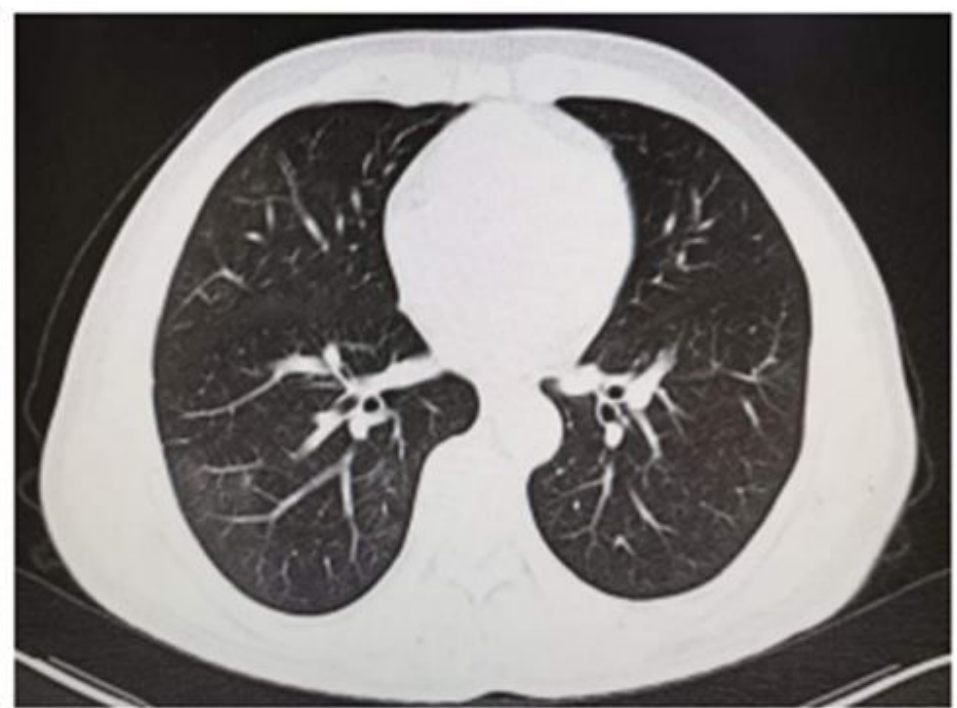

D 2020-02-05

Figure 3

Comparison of chest CT before and after treatment in case 2 . 


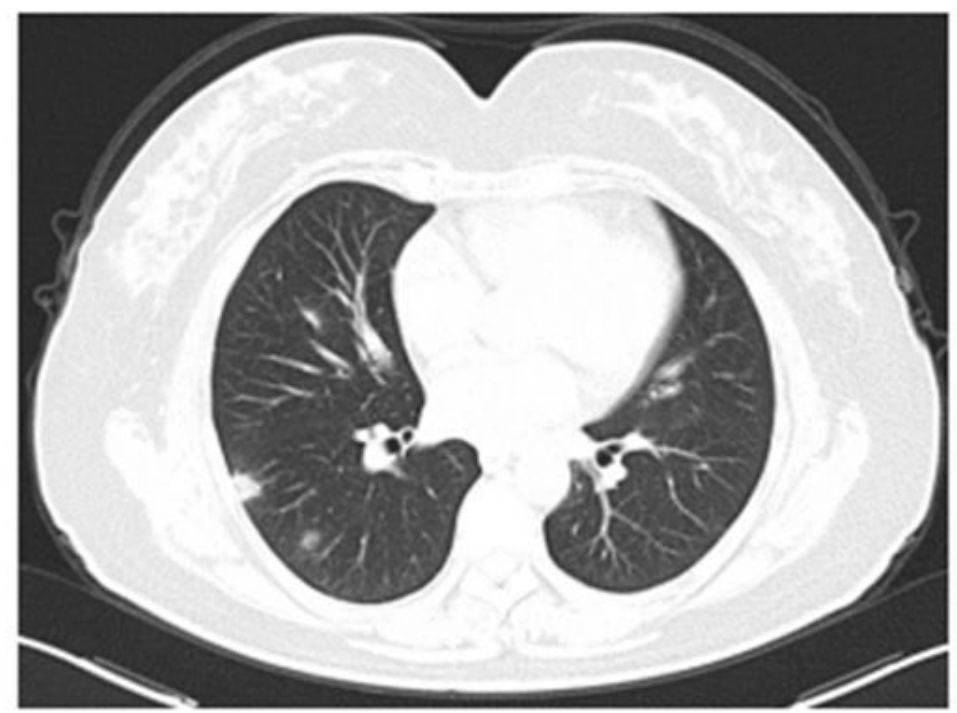

A 2020-01-30

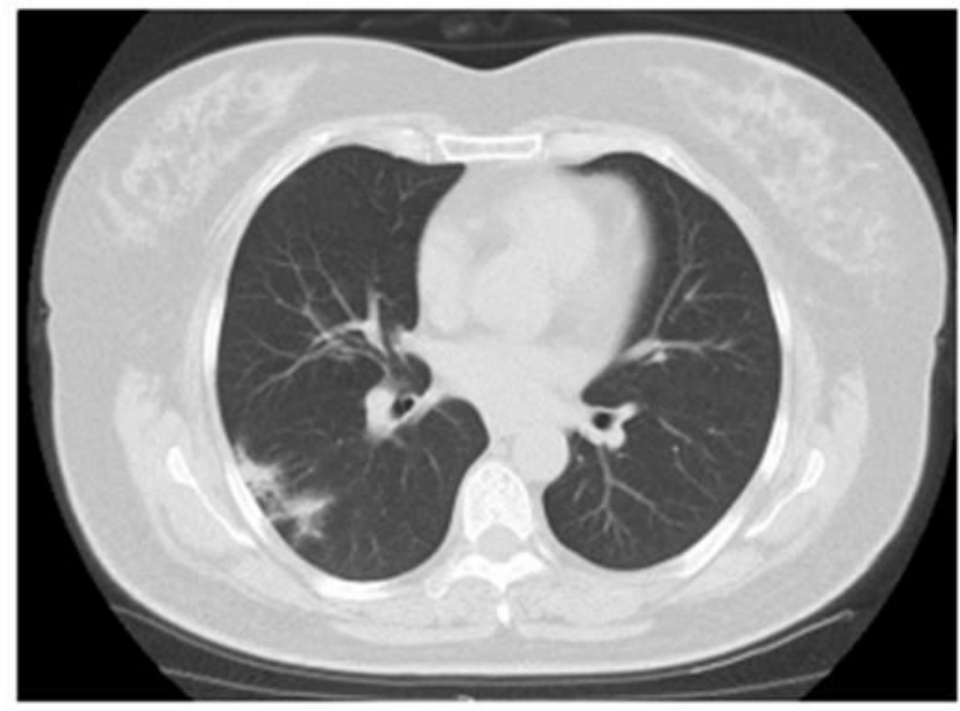

C 2020-02-11

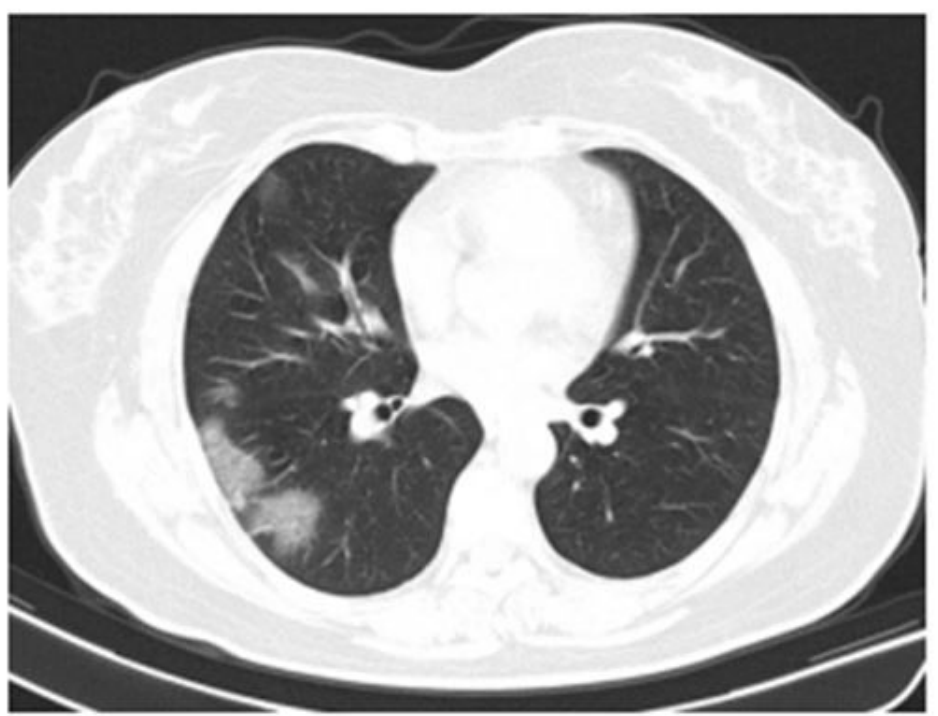

B 2020-02-05

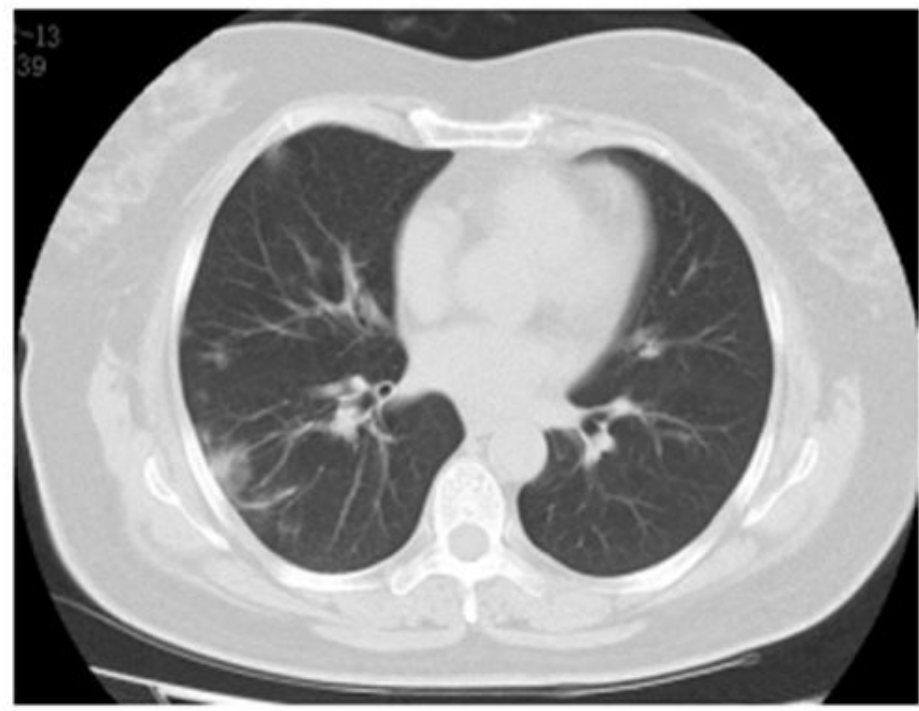

D 2020-02-13

Figure 4

Comparison of chest CT before and after treatment in case 3 . 


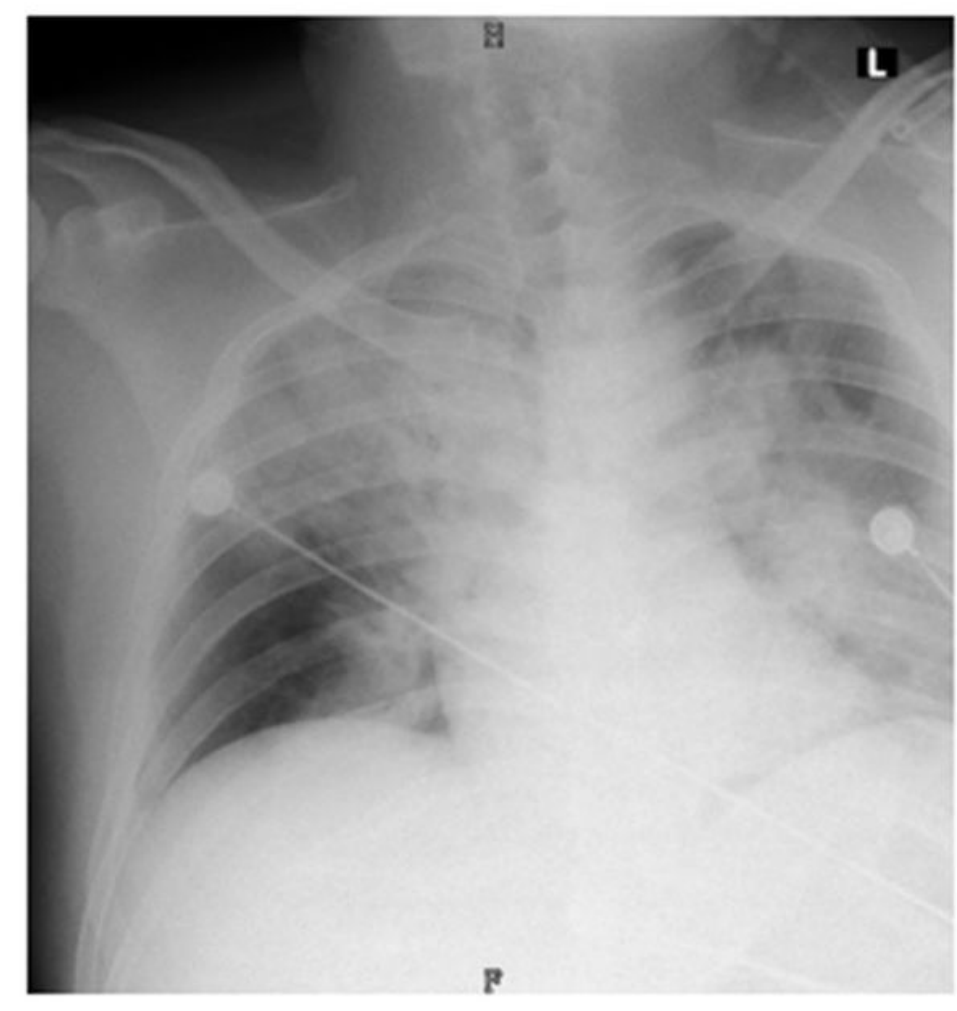

A $2020-01-31$

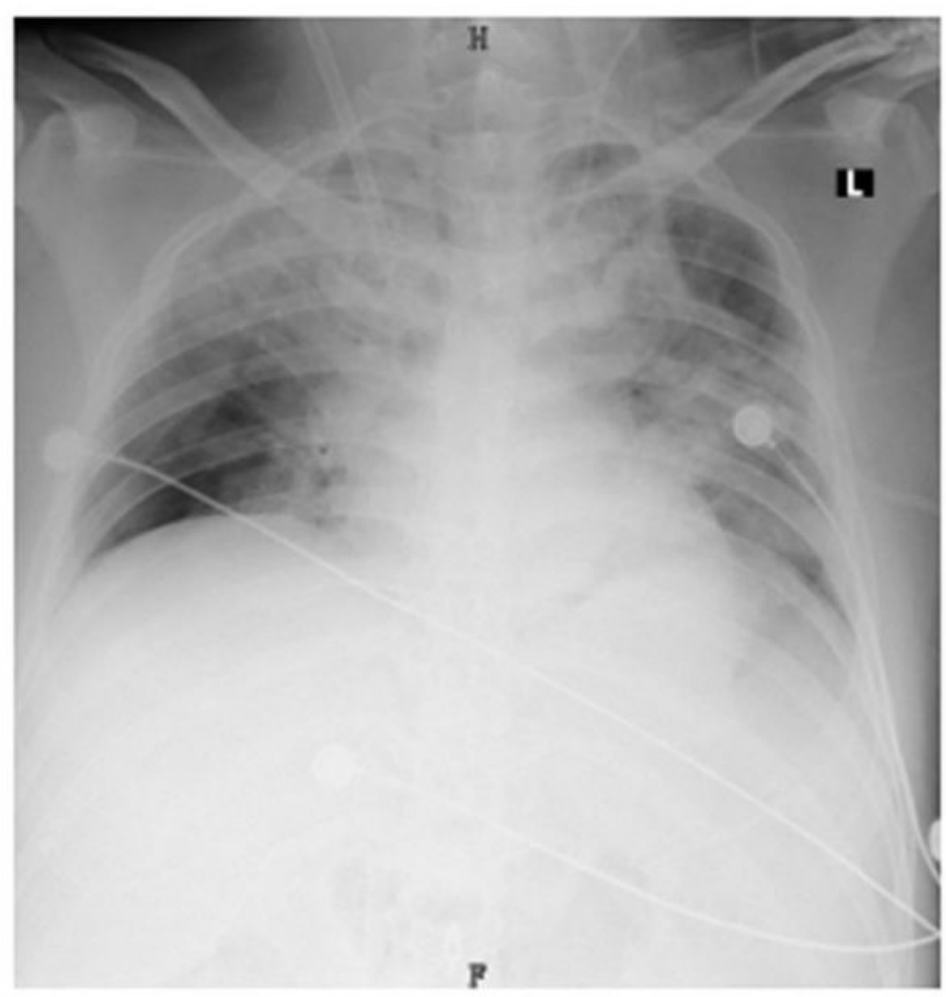

B $2020-02-03$

Figure 5

Comparison of chest X-ray before and after treatment in case 4 . 